heterosexual female. Sister/sister pairs must produce 33 per cent more young per pair for the same result. Although female/female pairs produce large clutches of eggs there is, however, no evidence that female/female pairs produce more young than male/female pairs, and they may in fact produce substantially less ${ }^{4.5 .10}$. Hence, males seem to provide such overwhelming advantages to their female mates that female/female pairings occur primarily when females are unable to pair with males ${ }^{11-13}$.

Connecticut Agricultural

MICHAEL R. CONOVER

Experiment Station, New Haven,

Connecticut 06504, USA

\section{Embryo and seed abortion in plants}

SIR-In asking "why do plants produce so many more ovules than seeds", Charlesworth ${ }^{1}$ and Wiens et al. ${ }^{2}$ have addressed an important issue. Wiens et al. attribute the high level of ovule abortion observed in many plants to genetic load, a factor also emphasized by Charlesworth in her commentary. We wish to point out that data presented by Wiens et al. are not consistent with their own hypothesis, and briefly we review alternative explanations $^{3-6}$ for ovule abortion.

Ovule abortion in plants occurs at two levels. In many species most flowers do not set fruits. Furthermore, in a number of species, all ovules within a flower do not set seeds. As Charlesworth points out, inbreeders generally have higher fruit/ flower and seed/ovule ratios, but exceptions are known. For example in the Mimosoideae, regardless of the breeding system, fruit/flower ratios are extremely low and seed/ovule ratios are very high? In other species with negatively skewed distribution of seeds within fruit ${ }^{6}$, the proportion of flowers and developing fruits that are aborted is very high as compared to the proportion of ovules aborted within the retained mature fruits. The exceptions provide insights into causes 'of ovule abortions and emphasize the need to consider separately the two levels of abortion, at least under some circumstances.

Wiens et al. deal with ovule abortion at the level of flowers. Only 2.5 per cent of flowers in Dedeckera eurekensis set fruits, though many more initiate the development of ovules. Such a low level of fruit set is not unusual in plants ${ }^{8,9}$. Wiens et al. attribute the low reproductive output in $D$. eurekensis to segregational load but also state that "Intraplant self-pollination (geitonogamy) may be common in $D$. edeckera, but inbreeding depression resulting from selfing in a normally outcrossed population is not a major factor contributing to the low seed set." As Charlesworth points out the segregational load hypothesis would predict strong inbreeding depression following selfing in outcrossing populations. Wiens et al. did not find the difference in fruit set between self- and cross-pollinated flowers to be statistically significant. The segregational load hypothesis also predicts abortions to occur randomly along the inflorescences. It is not known if the flowers are aborted at random in $D$. eurekensis, but in other species flower abortions are nonrandom (ref. 3 and J. H. Beach, personal communication). It is therefore premature to assume that segregational load is a major factor underlying abortion in $D$. eurekensis (and other species).

The abortion of ovules within fruits is briefly considered by Charlesworth ${ }^{1}$. Many species abort a variable number of their ovules after fertilization within developing fruits. Again the hypothesis of segregational load would predict random patterns of abortion. Once again, however, there is evidence for nonrandom abortion of seeds within fruits ${ }^{3,7}$. Genetic load due to recessive lethals also implies that the aborting embryos could never survive. In fact, aborting embryos within fruits, at least in some species, can be rescued if the dominant embryo is killed ${ }^{10}$.

Charlesworth does not discriminate between embryo and fruit abortion, both of which contribute to the lowered maturation of ovules (and hence lead to the question of why so many ovules). Such distinction has important implications because the pattern of abortion of embryos within a fruit ${ }^{6}$ and that of fruits within a plant $^{11,12}$ may be distinctly different. If embryo abortion is due to the genetic load, the retained fruits are expected to exhibit a normal distribution of seeds per fruit because the aborting embryos should be randomly distributed in all fruits. This however is not true and the seeds per fruit show varied but species-specific distribution patterns ${ }^{6}$. At least some of these patterns (for example, negatively skewed distribution of seeds within fruits) are not due to the embryo abortion; rather they are due to the seed-number-dependent selective abortion of pods ${ }^{8.11,13}$, which may or may not be dependent on genetic complement of the seeds ${ }^{12}$.

We have recently reviewed factors responsible for ovule abortion within developing fruits. Parent-offspring conflict over resource allocation and sibling rivalry can both lead to abortion of developing embryos ${ }^{6}$. The production of extra ovules may be a means of selecting embryos in the face of diversity of pollen genotypes reaching the stigmas ${ }^{4}$. Pollen competition has also been assumed to play a role in ovule abortion ${ }^{3,5.7}$. These hypotheses do explain the frequently observed correlations between the levels of abortion and mating systems. Unlike the genetic load hypothesis, these hypotheses also explain nonrandom abortions, correlations between patterns of abortions and seed dispersal and the low level of abortions in highly outcrossed taxa such as tree species in the Mimosoideae. But they do not explain postdevelopmental abnormalities in seed and seedlings observed by Wiens et al.; note, however, that evidence for such abnormalities is based on a sample size of only nine seeds ${ }^{2}$.

There is, therefore, little evidence that segregational load is a leading factor in the abortion of zygotes and embryos in natural populations of plants. Several other hypotheses provide alternative explanations for the patterns observed in nature. These hypotheses are based on reasonable genetic models and plausible developmental, physiological and ecological mechanisms. Until more data are available, therefore, segregational load should be considered as one of the least likely possible general explanations for ovule abortions.

Department of Biology,

K. S. BAWA

University of Massachusetts,

Boston, Massachusetts 02125, USA

S. G. HEDGE

K. N. GANESHAIAH

Department of Farm Forestry,

University of Agricultural Sciences,

GKVK Campus,

Bangalore 560065, India

R. UMA SHAANKER

Department of Crop Physiology.

University of Agricultural Sciences,

GKVK Campus,

Bangalore 560065, India

Charlesworth RePlies-I completely agree that genetic load is not a very likely explanation for ovule abortion in plants. I only gave the genetic load hypothesis serious attention, because it was emphasized in the paper ${ }^{2}$ on which I was commenting, and it was therefore necessary to explain the idea clearly enough to evaluate it critically. In my discussion of the hypothesis, I stated that segregational load (due to heterozygote advantage) cannot explain the data, and for mutational load that: "it seems unlikely that all this load will be expressed in the earliest stages of development so as to be detectable as reduced seed maturation. ..", and that the lack of evidence for strong inbreeding depression argues against this interpretation for the case of $D$. eurekensis that was under consideration.

As I also pointed out, if mutational load causes abortion "it should be random within fruits". The evidence for nonrandom abortion in the references provided by Bawa et al. is relevant to this point, as is the evidence for regular differences between species that I mentioned. Both these types of evidence show that abortion does not depend entirely on the genotypes 